\title{
JOURNAL OF INTELLIGENT SYSTEMS
}

\section{EDITOR-IN-CHIEF}

Hasan Fleyeh, Falun

\section{EDITORIAL BOARD}

Animesh Adhikari, Goa

Rageed Al-Hashemy, Baghdad

Manjunath Aradhya, Bangalore

Valentina Emilia Balas, Arad

Daniel Berrar, Tokyo

Çağatay Çatal, Istanbul

Sigrid Ewert, Wits

Narendra Kumar Gupta, Edinburgh

Eyke Hullermeier, Marburg

M.A. Jayaram, Tumkur

Cengiz Kahraman, Istanbul

Asad Khan, Clayton

Eunjin Kim, Grand Forks

Frank Klawonn, Wolfenbuettel

Philippe Le Parc, Brest

Pawan Lingras, Halifax

Vijay Kumar Mago, Thunder Bay

Zoran Majkic, Rome

Farid Meziane, Salford

Mariofanna Milanova, Little Rock

Elpiniki I. Papageorgiou, Lamia

Shaolin Qu, New York

Praveen Ranjan Srivastava, Pilani

Maytham Hassan Safar, Kuwait

Jeffery T. Walker, Little Rock

JingTao Yao, Regina

Siril Yella, Borlänge 
The aim of the Journal of Intelligent Systems is to publish articles in the field of intelligent systems. This field includes artificial intelligence, models and computational theories of human cognition, perception and motivation, as well as brain models, artificial neural networks, pattern recognition, neural computing and evolutionary computing.

ABSTRACTED/INDEXED IN Baidu Scholar · Cabell's Directory · Case · CNKI Scholar (China National Knowledge Infrastructure) · CNPIEC: cnpLINKer · Compendex · DBLP Computer Science Bibliography · Dimensions · EBSCO (relevant databases) · EBSCO Discovery Service · Engineering Village · Genamics JournalSeek · Google Scholar · Inspec · Japan Science and Technology Agency (JST) · J-Gate · JournalGuide · JournalTOCs · KESLI-NDSL (Korean National Discovery for Science Leaders) · Microsoft Academic · Naviga (Softweco) · Norwegian Register for Scientific Journals, Series and Publishers · PhilPapers · Primo Central (ExLibris) · ProQuest (relevant databases) · Publons · QOAM (Quality Open Access Market) $\cdot$ ReadCube $\cdot$ SCImago (SJR) - SCOPUS · Sherpa/RoMEO · Summon (Serials Solutions/ProQuest) · TDNet · TEMA Technik und Management · Ulrich's Periodicals Directory/ulrichsweb · WanFang Data · Web of Science: Emerging Sources Citation Index · WorldCat (OCLC)

ISSN 0334-1860 · e-ISSN 2191-026X

All information regarding notes for contributors, subscriptions, Open access, back volumes and orders is available online at http://www.degruyter.com/jisys

RESPONSIBLE EDITOR Prof. Hasan Fleyeh, Computer Engineering Department, School of Technology and Business Studies, Dalarna University, 79188 Falun, Sweden, E-mail: Editorial_JISYS@ degruyter.com

JOURNAL MANAGER Denis Fracalossi, De Gruyter, Genthiner Straße 13, 10785 Berlin, Germany Tel.: +49 (0)30 260 05-283, Fax: +49 (0)30 260 05-250, E-mail: denis.fracalossi@ degruyter.com

RESPONSIBLE FOR ADVERTISEMENTS Claudia Neumann, De Gruyter, Genthiner Straße 13, 10785 Berlin, Germany Tel.: +49 (0)30.260 05-226, Fax: +49 (0)30.260 05-264, E-mail: anzeigen@ degruyter.com

(c) 2019 Walter de Gruyter GmbH, Berlin/Boston

TYPESETTING Compuscript Ltd., Shannon, Ireland

PRINTING Franz X. Stückle Druck und Verlag e. K, Ettenheim

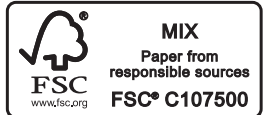




\section{Contents}

Aliya Fahmi, Saleem Abdullah, Fazli Amin and Asad Ali

Precursor Selection for Sol-Gel Synthesis of Titanium Carbide Nanopowders by a New Cubic Fuzzy

Multi-Attribute Group Decision-Making Model — 699

S. Pramod Kumar and Mrityunjaya V. Latte

Modified and Optimized Method for Segmenting Pulmonary Parenchyma in CT Lung Images, Based on

Fractional Calculus and Natural Selection -721

Seyed Mohammad Hossein Hasheminejad and Shabnam Gholamshahi

PCI-PSO: Preference-Based Component Identification Using Particle Swarm Optimization — 733

Vijay Kumar and Dinesh Kumar

Performance Evaluation of Modified Color Image Steganography Using Discrete Wavelet Transform — 749

Guiwu Wei and Mao Lu

Pythagorean Hesitant Fuzzy Hamacher Aggregation Operators in Multiple-Attribute Decision Making — 759

Pinjari Abdul Khayum and Reddy Pogu Sudheer Babu

Mitral Regurgitation Severity Analysis Based on Features and Optimal HE (OHE) with Quantification using PISA Method — 777

Xixi Wang, Farouk Yalaoui and Frédéric Dugardin

Non-dominated Sorting Genetic Algorithms for a Multi-objective Resource Constraint Project Scheduling

Problem -791

Weiying Guo, Yong Ji, Yong Luo and Yan Zhou

Substation Equipment 3D Identification Based on KNN Classification of Subspace Feature Vector - 807

Anil Kumar Dixit, Manmatha K. Roul and Bikash C. Panda

Mathematical Model Using Soft Computing Techniques for Different Thermal Insulation Materials — $\mathbf{8 2 1}$

Zhi-da Guo and Jing-Yuan Fu

Prediction Method of Railway Freight Volume Based on Genetic Algorithm Improved General Regression

Neural Network - 835

A. Hanif Halim and I. Ismail

Tree Physiology Optimization in Benchmark Function and Traveling Salesman Problem — 849

Amir M.U. Wagdarikar and Ranjan K. Senapati

Design and Development of a Multiobjective Cost Function for Robust Video Watermarking Using Wavelet Transform - 873

S. Sankar Ganesh, Pachaiyappan Arulmozhivarman and Rao Tatavarti

Forecasting Air Quality Index Using an Ensemble of Artificial Neural Networks and Regression Models — 893

Essam H. Houssein

Particle Swarm Optimization-Enhanced Twin Support Vector Regression for Wind Speed Forecasting — 905 